ARXIU D'ETNOGRAFIA DE CATALUNYA, N. 3, 1984

\title{
MEDICINA INTERVENCIONISTA VS. MEDICINA NATURALISTA: HISTORIA ANTROPOLÓGICA DE UNA PUGNA IDEOLÓGICA
}

\author{
Davydd J. GreEnwood \\ Center for International Studies. \\ Cornell University. \\ Ithaca, Nova York
}


En los Estados Unidos, donde la medicina científica ha sido organizada como parte de un cometido social general para hallar soluciones científicas a los problemas sociales, nos encontramos en medio de una crisis severa y decisiva. Por un lado, la admiración del público por los adelantos científicos y técnicos de la medicina no ha sido nunca más intensa que ahora. Simultáneamente, el descontento público con los que practican la medicina a nivel interpersonal, ha aumentado de manera vertiginosa.

La crisis actual se manifiesta bajo formas desgraciadamente bien conocidas. A pesar del aumento en la confianza hacia el médico científicamente entrenado, la medicina se practica bajo la constante amenaza de pleitos y querellas legales contra la negligencia médica. Por otra parte, y a menudo, los médicos encuentran grandes dificultades en conseguir que los pacientes sigan sus recomendaciones y consejos. Cuando un número cada vez mayor de jóvenes compiten académicamente para conseguir plazas de ingreso en las facultades de medicina, muchos médicos ya establecidos y en los años más productivos de su práctica escriben autobiografías en las que hablan de los horrores pasados en la facultad, las dificultades personales que encontraron al intentar ajustarse a la práctica de la medicina, las consecuencias del «desgaste» que acarrea la práctica diaria, y los problemas ocasionados por los médicos cuyas facultades han disminuido por razones diversas.

Nadie niega la existencia de esta crisis; es más, todo el mundo busca a la vez explicaciones y soluciones. Para ello se sirven de la siguiente perspectiva histórica: el principio del auge de la medicina científica en los Estados Unidos puede fijarse en 1910, año en el que se distribuyó el informe que el catedrático Abraham Flexner (1910) preparó bajo el patrocinio de la Fundación Carnegie. El desde entonces conocido como «Informe Flexner» es un documento exhaustivo y revelador que incluía informes sobre las visitas de inspección efectuadas a todas las facultades de medicina existentes entonces en los Estados Unidos, un análisis del curriculum y la planta física de cada una, y la confección de una serie de recomendaciones para la reforma de la educación médica.

El núcleo principal del «informe Flexner» consiste en la propuesta de unificar de modo coherente las ciencias biomédicas, la experiencia práctica 
en los hospitales, y la educación/entrenamiento médico. La aportación de Flexner a las ciencias y a la tecnología médicas está fuera de dudas. Sus críticas a las facultades de medicina existentes entonces iban dirigidas sobre todo a las deficiencias científicas y técnicas. Creía que el divorcio entre la ciencia y la práctica médica conducía y conduce a un nivel muy bajo de profesionalidad con desastrosas consecuencias sociales. Y muchas de sus recomendaciones se llevaron a la práctica.

Los observadores e investigadores de la crisis médica contemporánea, sin embargo, tienden a ver este doble aspecto de ciencia y de tecnologia como una espada de dos filos y como la causa misma de la crisis. En su libro Medical Nemesis (1976), Ivan Illich, por ejemplo, carga el peso de la culpa ne el poder enajenador de la tecnología moderna. En un análisis más complejo y ponderado, Stanley Joel Reiser, por su parte, define la crisis con mayor precisión:

Los expertos médicos que se interesen en hallar los factores sociales y emocionales integrantes del proceso de la enfermedad, deben seguir con la advertencia que han venido haciendo a sus colegas desde principios de este siglo; es decir, que la cura de la enfermedad requiere más que el sanar las partes del cuerpo... Y si esta creencia se mantiene en el futuro, tal vez pueda acortarse la distancia que la tecnología ha fomentado entre médico y paciente. (Reiser. 1978: 230-231).

Arthur Kohrman, médico y académico, atribuye parte del problema al distanciamiento típico del entrenamiento médico entre ciencias y humanidades. Aboga por la restauración de las relaciones entre la medicina y los estudios de letras:

No aconsejo una vuelta a los estudios clásicos tradicionales de las humanidades basadas en la nostalgia o en un presunto valor abstracto; tampoco propongo, como ha hecho recientemente Lewis Thomas, que todos los estudiantes de medicina deban estudiar griego clásico. Lo que sí propongo para el curriculum de la medicina es una combinación de formas antiguas y tecnología moderna; una vuelta a las humanidades que es necesaria, posible y oportuna. Sabemos que en los departamentos de humanidades de nuestras universidades se estudian las imágenes, las fábulas, las visiones míticas, las luchas contra el destino, la suerte, en resumen, el examen de los límites de las posibilidades humanas. Nuestros estudiantes, y las promociones venideras deben incorporar estos recursos de las letras, dado que todos reconocemos que la ciencia y su sierva ayudante, la tecnología, no pueden por sí mismas resolver los dilemas humanos fundamentales y eternos. Podemos, pues, formar nuevas generacio- nes de médicos para los cuales los arcanos provocarán respeto reverencial, lo desconocido despertará la curiosidad y agitación intelectuales: médicos que podrán así comprender un poco mejor a las personas que tienen a su cargo. (Kohrman, 1979:16).

Un líder influyente del movimiento "holístico» en medicina, Kenneth Pelletier, razona que la superioridad de la medicina sobre otras ramas de la ciencia nos ha conducido a un dualismo innecesario entre los métodos materialistas analíticos y los métodos «holísticos» sintéticos.

«Fue en la no lejana fecha de 1858 cuando la medicina se trasladó del ámbito de los debates teológicos y filosóficos al ámbito de la ciencia racionalista... Tal método positivista-racionalista nos há permitido hacer progresos evidentes y fue en su tiempo consistente con las corrientes científicas y filosóficas de los siglos dieciocho al veinte. Es sin embargo cada vez más obvio que limitar todas las investigaciones y aplicaciones clínicas a tal paradigma dualista no es defendible y resulta además anacrónico para la ciencia y la filosofía del siglo veinte. (Pelletier, 1975:25).

Una gran mayoría de los investigadores que estudian la crisis de la medicina moderna ha elaborado explicaciones semejantes a las arriba citadas. $\mathrm{Su}$ argumento fundamental es sencillo y se puede resumir así:

El auge de la medicina científica ha traido consigo una ruptura innecesaria entre los elementos técnico-científicos, requeridos en el tratamiento de los pacientes, y los métodos humanistas e integradores que se suponían connaturales a la medicina antes de la moderna obsesión por la ciencia aplicada y la tecnología.

Aunque hay en esta perspectiva elementos que tratan de actitudes que es innegable que prevalecen en la medicina, opino que estos argumentos son incorrectos. En primer lugar se basan en una falsa imagen histórica de la medicina. En segundo, todos estos argumentos comparten el mismo defecto al tratar la medicina moderna como una práctica uniforme, siendo como es evidente que la medicina moderna es enormemente diversa y que la ciencia y la tecnología, por otra parte, no se distribuyen de forma homogénea ni del mismo modo entre todas las especialidades. En tercer lugar, aunque esta manera de concebir la historia de la medicina encierra una crítica legítima, dado que nos acusa de habernos valido demasiado de la ciencia y la tecnología, la crítica es básicamente irónica y no desinteresada. De hecho, es casi una alabanza al poder que tienen la ciencia y la tecnología para transformar nuestra sociedad. Contiene además elementos de corte freudiano sobre la condición humana al sugerir que nuestras propias necesidades emocionales 
y capacidades intelectuales nos conducen a un sentimiento de infelicidad colectiva.

En cuarto y último lugar, las críticas antes mencionadas separan el auge de la tecnología médica moderna del contexto político-económico dentro del cual se ha venido desarrollando. Tratando a la ciencia y la tecnología como la causa misma, estas perspectivas desvían nuestra atención de los conflictos sociales implicados en el desarrollo de la profesión médica y en la distribución de la ayuda sanitaria en nuestra sociedad.

En esta presentación voy a centrarme solamente en dos de los problemas que plantea esta visión de la historia de la medicina. En primer lugar quiero resaltar la complejidad histórica de la relación entre la medicina y otras ramas de la ciencia. En segundo lugar, presentaré ejemplos para ilustrar la necesidad de considerar la diversidad de las especialidades médicas al hacer un análisis de la antedicha relación ciencia/medicina. Para terminar voy a proponer un proyecto de acercamiento antropológico, esbozando la forma que tal perspectiva antropológica puede tomar.

\section{El origen de estas investigaciones}

Hace aproximadamente seis años inicié investigaciones con el propósito de trazar la historia de las numerosas visiones de la naturaleza humana que de una u otra forma se basan en un determinismo biológico (Greenwood, MS). Juzgué de especial importancia evaluar el impacto del darwinismo sobre estas perspectivas deterministas, pues he llegado a la conclusión que el modelo evolucionista de Darwin no ha sido incorporado de ninguna manera en las teorías deterministas contemporáneas. Tomé como punto de partida y comparación para este proyecto el examen de las perspectivas predarwinianas sobre la naturaleza y sobre la naturaleza humana; estudio que naturalmente hay que iniciar con un examen detenido de la influencia general de los textos hipocráticos y galénicos. Una larga y enriquecedora lectura de esos textos me llevó a la conclusión de que un acercamiento antropológico a estos problemas requiere una comprensión sustancial de la manera como la tradición humoral concebía la condición humana.

Para investigar la aplicación concreta y diferenciada de las teorías humorales desde un punto de vista antropológico, pocos lugares son más idóneos que unas instituciones en las cuales las ideas humoralistas han continuado y persisten hasta el momento: los balnearios. Con esta idea empecé una investigación que aún no se ha completado y que se centra en el estudio de algunos balnearios españoles, en el análisis de las teorías hidrológicas del trata- miento de clientes, y la lectura de una amplia literatura sobre las virtudes de las aguas que se encuentran en distintas localidades de este país. Como parte de aquella investigación estudié algunos documentos de historia de la medicina española en los cuales las teorías humorales tuvieron un importante papel.

Aunque pienso escribir sobre esas investigaciones en un futuro próximo, encontré entre los documentos históricos los trabajos de dos figuras de la Ilustración española que considero pertinentes y representantes de la contienda ideológica analizada en la discusión que nos ocupa. Se trata del padre Benito Jerónimo Feijoó y de don Diego de Torres Villarroel. Críticos acerbos de la medicina "científica» de su época, ambos miraron con favor los tratamientos humorales. Las citas que siguen resumen sus ideas:

Los médicos saben poco de la curación de los enfermos; pero nada saben, ni aun pueden saber en particular de el régimen de los sanos, por lo menos en quanto a comida y bebida. (Feijoo, 1724-39, Tomo I: 142).

Pretendo, pues, que no solo el Médico pueda serlo [médico de sí mismo] respecto de si proprio [sic], quando esté enfermo mas qualquiera enfermo puede, y debe serlo en parte, respecto de si proprio [sic]. (Feijó, 172438, Tomo IV: 70 ).

...todos los sujetos chicos y grandes del mundo, sean naturales o artificiales, han de sostener en sí los cuatro elementos: Luego todos, sobre poco más o menos, han de soltar una misma virtud e, introducidos en nuestros cuerpos, los nutrirán, purgarán, darán sueño y vigilia, y los inclinarán a las demás buenas o malas, sanos o enfermas, operaciones con que notamos, alegres y afligidos, mozos y viejos, vivos y muertos, a los cuerpos humanos. Pues todo lo criado concurre a darlos salud, enfermedad, tristeza, gozo, vida y muerte. (Torres Villarroel, 1970: 180).

Todo mi cuerpo es una portátil enfermería de humores. Estoy enfermo. $\mathrm{Y}$ deshauciado por la naturaleza. Estoy enfermo. Por eso me he reído yo de la medicina porque es tan loca, que presume dar salud al hombre mortal. (Torres Villarroel, 1794-1799, Tomo III: 341).

La medicina que se estudia en las Universidades, es un vocabulario de términos que suenan bien y hacen mal, valen poco y cuestan mucho, y nos venden sus aprehensiones $\tan$ caras, que nos suelen costar la vida. (Torres Villarroel, 1794-1799, Tomo IV: 200).

Lo que nos importa está claro para todos: es ciencia propia la de las almas, y en ella es solo docto el que conferencia consigo. El estudio de la Medicina empieza por el conocimiento de nuestra arquitectura y economía: más cerca está mi cuerpo de mi consideración que el de los otros... sin más trabajo que el prudente aprecio de las voces y gritos que nos da la razón natural, sabremos conocer nuestros achaques y alivios mejor que el Médico: y con mayor claridad y menos costa hemos de cuidarnos nosotros que él. (Torres Villarroel, 1794-1799, Tomo IV: 7-9). 
Debo hacer notar, quizás innecesariamente, que Torres pertenece al grupo de científicos. Médico y doctor de universidad, fue catedrático de matemáticas de la Universidad de Salamanca. Escribió muchos tratados para estimular la educación científica del público, e incluso varias guías y manuales de medicina. Torres veía la medicina científica de su époea como dominada por la ciencia mecanicista y abstracta que no tomaba en cuenta las necesidades de la persona como entidad ni llegaba a entender la salud como un fenómeno integrador.

La crítica de la Medicina hecha por Torres tiene tanto en común con las críticas contemporáneas de la medicina científica, que debemos preguntarnos sobre la validez y novedad de estas perspectivas críticas modernas. Parece ser que en el Occidente la medicina se ha caracterizado durante siglos por un conflicto entre dos visiones fundamentalmente opuestas: una intervencionista y técnica y la otra, naturalista y sintetizadora.

No implico con esto que la medicina no sufriese cambios entre el siglo XVIII y el presente; por el contrario, el conflicto entre la medicina científica y la medicina naturalista ya existía antes de la creación de la ciencia médica moderna. Puede pensarse que la Medicina científica de la época de Torres no era científica en ningún sentido de la palabra. Rechazo absolutamente tal idea.

La ciencia no es un fenómeno exclusivo de nuestra época. Aunque nos parezcan modestos los descubrimientos y las técnicas de los siglos anteriores en comparación con lo que tenemos ahora, siguen siendo descubrimientos científicos. Basados en una combinación de observación, experimentos y razonamientos, los descubrimientos científicos de nuestros antepasados a menudo se expresaron en forma de declaraciones falsificables. Aun las historias de la medicina más tradicionales señalan la existencia de la ciencia médica en este sentido.

Habiendo descubierto esto volví a los textos de la historia de la medicina con la esperanza de encontrar alguna confirmación de lo que había ya visto. Sin embargo, la literatura a este respecto es desgraciadamente decepcionante. Una excepción de interés la constituye una historia monumental y poco leída de la Medicina escrita recientemente por Harris Livermore Coulter. Publicado en tres tomos con el título de Divided Legacy (Coulter, 1973, 1975, 1977), su tema principal es que la herencia de la medicina occidental ha estado siempre dividida entre estas dos visiones. Coulter entiende la historia de la medicina como un conflicto continuo entre estas dos tendencias. Ofrece una documentació realmente impresionante y, a mi entender, convincente; sin embargo, Coulter tiene su propia agenda polémica. Quiere que la medicina científica reconsidere la tradición homeopática de Samuel Hahne- mann. Insiste Coulter en que las teorías de Hahnemann no recibieron la debida atención porque la medicina moderna no llegó a reconocer que la medicia naturalista también puede ser científica. Los libros de Coulter no se han leído mucho ni tampoco han sido aceptadas sus perspectivas. Muchos médicos y académicos aparentemente prefieren tratados más cortos que cargan los problemas contemporáneos sobre la ciencia y la tecnología.

Además de la obra de Coulter hay unos cuantos libros que tratan de la llamada «medicalización" de la sociedad y de la manipulación de la salud y la enfermedad como instrumentos del control social e ideológico. El nacimiento de la clínica, de Michel Foucault (1973), y The explotation of illness in capitalist society, de Waitzkin y Waterman (1974), tocan este tema desde varios puntos de vista. Estas perspectivas merecen atención porque hacen hincapié en la hegemonía ideológica de la ciencia moderna e insisten en basar el análisis de la medicina moderna en un contexto cultural, social e histórico específico.

Lo más destacable de esta polémica es la extraordinaria continuidad del conflicto entre la medicina intervencionista y la medicina naturalista. Esta continuidad queda por explicar. Necesitamos una convincente combinación de análisis sociales e ideológicos que nos pueda proporcionar una comprehensión de las fuentes de este continuo conflicto.

Como hipótesis de investigación antropológica sugiero que la tensión entre la medicina intervencionista y la medicina naturalista forma parte intrínseca de la relación entre médico y paciente según se efectúa el mundo occidental. Esta tensión renace constantemente porque forma parte de la estructura básica de la medicina occidental.

En el análisis que sigue, por lo tanto, me propongo examinar la relación entre médico y paciente desde un punto de vista antropológico para llegar a las raíces de esta tensión. Paso luego a demostrar cómo este tipo de análisis puede ayudarnos a interpretar el trayecto histórico de la medicina moderna. Esta presentación será necesariamente muy esquemática, por las restricciones de tiempo y porque las investigaciones de las cuales entresaco estos datos aún no han terminado. Empiezo con un modelo abstracto de la relación entre médico y paciente y a continuación relaciono estos modelos con unos análisis concretos de la diversidad de las experiencias en las distintas especialidades médicas en los Estados Unidos.

Puede parecer que lo que describo se limita a los Estados Unidos. No es así. La tradición médica occidental es efectivamente eso: occidental. La historia institucional y cultural de cada país (y aun de las regiones dentro de un país) difieren, pero dentro de un campo mayor que llamamos la medicina occidental Quieio hacer en el futuro comparaciones rigurosas de los 
sistemas médicos de los Estados Unidos, España y otros países occidentales, pero de momento voy a ocuparme de una perspectiva muy general que a grandes rasgos se aplica a todos. En mi análisis los datos etnográficos sobre la medicina científica moderna se limitan solam nte a los Estados Unidos, y tendré mucho gusto en recoger y discutir dat' $s$ comparables sobre la medicina científica moderna en España.

Lo que presento a continuación, de forma muy breve, es un ideal type en el sentido weberiano estricto. Consiste en $l_{1}$ contraposición de una pareja de perspectivas abstractas y polarizadas que se refieren a relaciones sociales y culturales y que se emplean para ayudarnos a interpretar los procesos históricos concretos. Esto no quiere decir que estos ideal-types sean directamente observables. Etnográficamente incorporan una abstracción de las tendencias básicas dentro de los datos específicos. La prueba apropiada de tales ideal-types es su utilidad en el examen de datos etnográficos específicos y de procesos históricos también específicos.

Debo aclarar desde el principio que deduzco un grupo de conflictos médicos de un análisis sincrónico de la lógica de las relaciones entre médico y paciente en nuestras sociedades de Occidente. Tal método encierra riesgos, especialmente porque intento llegar a una interpretación histórica de los conflictos médicos.

Este método se justifica de dos maneras. En primer lugar nos proporciona resultados analíticos nuevos y útiles. En segundo, una idealización popular de la relaicón entre médico y paciente parece haber gozado de una notable continuidad en el mundo occidental. El Juramento Hipocrático, tan prominentemente exhibido en las consultas médicas de los Estados Unidos, es una idealización, pero, por lo visto, una idealización que capta la esencia de lo que quisiéramos ver como relación entre médico y paciente.

\section{La relación entre médico y paciente: la medicina intervencionista}

Una de las características fundamentales de la relación entre médico y paciente en la medicina intervencionista es ia falta de simetría en las experiencias de esta relación. Es aquí donde debemos iniçiar el análisis.

La conciencia de su enfermedad que adquiere el pacientc empiezs como un acontecimiento puramente personal. La enfermedad en general se experimenta como una alteración del nivel normal de funcionamiento, un cambio que es privado, a menudo progresivo, y que llega a ser una amenaza cuando su seriedad lo proyecta al plano familiar y finalmente al escrutinio público. No podemos enfatizar demasiado el hecho de que el paciente expe- rimenta la enfermedad a la vez como una alteración de su funcionamiento normal y como una forma de dependencia social en la cual los detalles más intimos de su existencia privada se trasladan a dominio público. Esto crea unas formas de dependencia muy elaboradas y complejas para todos los que guarden relaciones con el enfermo, incluso el médico a quien se ha recurrido.

Desde este punto de vista es justo decir que el paciente se encuentra en un estado de riesgo al visitar al médico. El peligro proviene de la enfermedad en sí y de la dependencia que la enfermedad ha creado para él. El paciente pierde una parte importante de sus prerrogativas de adulto responsable en el acto mismo de buscar el tratamiento médico.

Desde el punto de vista del paciente, el médico es un elemento único en su vida. La llegada a la consulta del médico es el final de un largo proceso en el cual el control autónomo sobre su vida se ha perdido o por lo menos se ha comprometido bastante. El paciente no se piensa a sí mismo como uno de los muchos pacientes del médico, aunque todos los pacientes se dan cuenta de que hay muchos otros todos los días. El paciente se acerca al médico con la esperanza de que a cambio de la nueva dependencia establecida va a encontrar explicaciones y soluciones a sus dilemas.

El paciente quiere saber qué es lo que tiene. Es de absoluta importancia que el médico llegue a un diagnóstico claro y que sea intelectualmente satisfactorio para el paciente. El paciente también quiere saber curar su mal o por lo menos cómo llegar a controlarlo para poder recuperar su funcionamiento autónomo. Es por lo tanto necesario elaborar un régimen convincente de tratamiento.

De importancia más básica todavía, el paciente quiere saber cómo y por qué él o ella ha sido invadido por la enfermedad, especialmente si es una enfermedad que le amenaza con la muerte. Aquí la falta de simetría entre las perspectivas del médico y del paciente es muy evidente. Aunque el médico pueda responder a la pregunta "¿cómo es que me he puesto enfermo? ", los médicos no se ven obligados a contestar al "porqué» de la enfermedad.

El paciente ha experimentado la enfermedad como una dependencia y deterioro progresivos que necesariamente ha de tener causas tanto morales como físicas. Es casi imposible que los pacientes seriamente afligidos no busquen explicaciones morales a sus enfermedades. «¿Por qué sufro yo?» «¿Qué he hecho yo para merecer esto?» A menudo éstos son los interrogantes más importantes en la conciencia del paciente. No conocemos como antropólogos ninguna sociedad que interprete los males que afectan a sus miembros como resultado de acontecimientos fortuitos. Como intelectuales racio- 
nalistas intentamos convencernos de que la búsqueda de causas morales para explicar sucesos fortuitos es una forma de superstición. Pero es evidente que los humanos no aceptamos la idea de que las cosas terribles nos pasan sin culpa ni razón aparente ninguna. La búsqueda de la explicación moral de la causa y sentido de la enfermedad es una característica común a todas las sociedades. Una consecuencia de esto es que el diagnóstico del médico y sus recomendaciones sobre el tratamiento a menudo son interpretados por el paciente de una manera bastante más compleja de lo que piensa el médico. No solamente se incorporan los síntomas físicos y los procesos fisicos del tratamiento, sino también el juicio del paciente sobre la cuestión de si el "castigo" es proporcionado al «crimen" de la enfermedad y si, por lo tanto, va a conducir a una vuelta a la independencia física y al equilibrio moral. Si el tratamiento se desarrolla con éxito, el paciente experimentará el fin del episodio de la enfermedad como un aumento de independencia y una vuelta al dominio privado y moralmente aceptable como miembro legítimo de la sociedad. Guarda además la esperanza de quedar con pocos estigmas que perduren.

La experiencia del médico, en la mayoría de los casos, es bastante distinta. Es el paciente el que va a ver al médico. En la medicina estadounidense ya casi no hay médicos que hagan visitas a domicilio. Explican los médicos que esto es resultado de la necesidad de tener a mano la tecnología moderna y sus laboratorios, cosas que no se pueden transportar con facilidad.

Mientras el paciente se aproxima al médico con un «status" de dependencia, al médico le llega el paciente como uno de los muchos episodios que tiene que tratar durante un día lleno de episodios semejantes. Al paciente se le ve menos como persona que como un problema por solucionar, $\mathbf{y}$ en ocasiones un fracaso en potencia para el médico.

El complicado proceso de elaborar el historial y el diagnóstico clínico implica la descripción de síntomas que tienen que colocarse dentro de un esquema clasificatorio. Esto forma parte del proceso adivinador de la medicina. Los sintomas se tratan como signos de las entidades subyacentes que constituyen la enfermedad. Distintas enfermedades pueden causar sintomas semejantes. A menudo este proceso de diagnóstico es muy difícil. Muchas veces ocurre que el médico se da cuenta perfectamente de que el paciente sufre algún mal importante, pero no lo puede captar en un diagnóstico claro. La credibilidad y competencia profesionales del médico se ven comprometidas muy rápidamente, porque es deber del médico clasificar síntomas y diagnosticar las enfermedades sin titubear. El paciente tiene esta expectativa y esperanza, y los médicos a menudo han proclamado que su ciencia tiene esta capacidad. El intento fracasado de diagnosticar una enfermedad de manera convincente se percibe como una especie de descenso en su «status» como médico.

Una vez que se decide el diagnóstico, el médico tiene la obligación de articular un pronóstico que tiene como función el estructurar las expectativas del paciente frente al curso de su enfermedad. Este pronóstico puede ser más o menos elaborado, y el médico lo mide de acuerdo con su evaluación de la capacidad de comprensión que percibe en su paciente. A menudo los médicos no explican el pronóstico completo; prefiriendo pasar a los pacientes, paso por paso, por el proceso curativo.

A continuación el médico tiene que emprender la confección de un régimen de tratamiento que sea coherente con la clasificación, diagnóstico, y pronóstico, y capaz de estimular la cooperación del paciente. Los médicos saben muy bien que si algún aspecto de la clasificación, diagnóstico, pronóstico o tratamiento no convence al paciente, la probabilidad de conseguir la colaboración del paciente en el régimen de tratamiento es muy baja. Los médicos juzgan que en general el nivel de obediencia entre sus pacientes es menor del $50 \%$. El fracaso en el intento de conseguir la cooperación de sus pacientes se percibe como un desafío a su autoridad o como señal de la falta fundamental de educación y racionalidad entre sus pacientes.

Si nos pusiéramos a examinar los problemas morales del médico, veríamos que son problemas centrados en la superioridad científica del médico sobre sus pacientes. El médico es un científico que tiene como obligación la clasificación, la diagnosis y el tratamiento de las enfermedades. El fallo en cualquiera de estas obligaciones se experimenta como un fracaso muy profundo. Fracasos de este tipo, o sencillamente una serie de problemas médicos insolubles, fácilmente pueden llevar a un médico a una crisis de su confianza en sí mismo como médico. Un índice tolerable de éxito y de cooperación por parte de sus pacientes es necesario para la confianza del médico en sí mismo y para mantener su sentido del valor moral como persona.

Merece la pena subrayar el hecho de que el médico no sienta ninguna obligación de explicar el porqué un paciente en particular sufre de alguna enfermedad específica. El médico se interesa por la identidad de la enfermedad, y como mucho por cómo llegó el paciente a enfermarse desde el punto de vista epidemiológico. La necesidad que experimenta el paciente de obtener respuesta a la pregunta «por qué» respecto a su enfermedad no es considerada en general por el médico científico como parte de su obligación profesional.

Complicadas relaciones de jerarquía y de igualdad, por lo tanto, atraviesan las relaciones entre médico y paciente. El médico tiene una reconocida superioridad con relación al paciente, pero la enfermedad y el comporta- 
miento del paciente constituyen una amenaza seria para el médico. Aunque el paciente se encuentra en una posición social inferior a la del médico, la enfermedad o el paciente mismo pueden resultar intratables y pueden ser un obstáculo en el sentido de valor y satisfacción que el médico busca en la prosecución de su vocación médica. Y, finalmente, el paciente se encuentra totalmente sin apoyo al intentar explicarse por qué le ha atacado una enfermedad que es causa de tan grandes alteraciones en su vida.

\section{La relación entre médico y paciente: la medicina naturalista}

En la modalidad naturalista de la medicina, aunque se encuentran presentes algunos de los mismos elementos, la relación entre médico y paciente es muy distinta. Para empezar, la tradición naturalista evita la conceptuación de la enfermedad como una entidad. La enfermedad y la salud forman parte de la persona; el único y complejo equilibrio entre las partes lo constituye la innegable individualidad de cada ser humano, incluso la del médico. Todos los seres humanos combinan la salud y la enfermedad. La medicina es un intento de restaurar el equilibrio natural de cada individuo, cualesquiera que sean las bases de aquel equilibrio.

Desde esta perspectiva el paciente no experimenta la enfermedad como algo que le invade desde fuera. Al contrario, es la expresión de las fuerzas internas que se han desequilibrado: la potencialidad de sufrir un desequilibrio tal existe en todas las personas. Una consecuencia es que la transición inicial a la enfermedad no se experimenta como una invasión externa, contaminadora del individuo como persona privada. Sin embargo, la enfermedad que padece puede robarle al individuo la capacidad de gobernarse independientemente. Por lo tanto la misma transición de la independencia a la dependencia forma parte del cuadro de la enfermedad en esta tradición.

Cuando un paciente se entrega al médico, esta entrega se efectúa de una manera muy distinta a lo que se ha visto en la medicina científica. El médico mismo no se encuentra libre de la enfermedad desde el punto de vista naturalista porque todos los individuos se caracterizan por un equilibrio entre fuerzas positivas y negativas varias. El médico no se encuentra en un plano distanciado del paciente. Su humanidad común les proporciona problemas semejantes, aunque sus respectivos equilibrios son muy distintos. La autoridad del médico se basa no en la ciencia de por sí, sino en su experiencia y sus conocimientos. Como médico práctico, él aplica los conocimientos que tiene de las substancias y las alteraciones ambientales que pueden conducir a la restauración del equilibrio del paciente. La individualidad del paciente nunca se pierde de vista en este modelo porque no se busca una vuelta al estado estadísticamente normal, sino al estado de funcionamiento original del individuo.

No se dede enfatizar la importancia de considerar al paciente como un ente médico-moral que sufre un desequilibrio en vez de considerarlo como un individuo invadido por una enfermedad. Parece que los interrogantes sobre el por qué de un estado de enfermedad en particular son menos urgentes para el paciente en esta tradición. No es porque el problema médico en sí mismo no les interese a los pacientes, sino porque el concepto general de la vida como una combinación de salud y enfermedad, del equilibrio y el desequilibrio, hacen que un episodio particular de enfermedad sea una parte más íntegra del proceso general de la vida. La enfermedâd no es una ruptura, una invasión, una entidad extraña que de repente vive dentro de la persona; la enfermedad es una parte de la vida humana vista como una totalidad. Esto no quiere decir, claro está, que los pacientes estén contentos al encontrarse enfermos, especialmente si se trata de enfermedades serias. Pero la presión sobre la relación médica para porporcionar una comprensión general de «por qué» ha afectado una enfermedad específica a un individuo en particular disminuye de forma notable.

Desde el punto de vista del que practica la medicina naturalista, la experiencia médica es también distinta de la que se encuentra en la medicina cientifica. La premisa inicial no refleja una actitud de superioridad sobre los pacientes, sino que se refiere a su mayor experiencia y sus conocimientos. Considera a cada paciente como uno entre los muchos episodios que requieren tratamiento médico, exactamente como en el caso del médico intervencionista. Aunque el concepto naturalista inclina al experto en esta medicina a pensar en cada paciente en términos muy individuales, el paciente todavía se presenta al médico como un desafío, como un rompecabezas, como una amenaza a su competencia médica. La clasificación, el diagnóstico, el pronóstico y el tratamiento tienen que ser elaborados utilizando una materia básica que es compleja y refractaria.

Sin embargo, el proceso de clasificar, diagnosticar y tratar están aquí influidos por el concepto de la individualidad fundamental del estado de salud de cada paciente. Hay menos intentos de tipificar los estados de la enfermedad porque cada uno debe ser, en la medida posible, único al formar parte de la vida de un paciente determinado. La misma individualidad del paciente que crea dificultades en el diagnóstico es vista por el práctico naturalista como fuente de datos para diseñar el tratamiento idiosincrático e individual que se tendrá que adoptar. 
Aunque no existen todavía datos seguros con respecto a la colaboración de los pacientes, parece ser que ésta es en general mucho menos problemática en la medicina naturalista. En primer lugar, los pacientes a menudo han recurrido al médico naturalista después de haber tenido una experiencia poco satisfactoria con la medicina científica. Por lo tanto, están convencidos y determinados a seguir este método alternativo. Por otra lado, como los tratamientos tienden a ser menos radicales y el diálogo entre médico y paciente es más igualitario, la colaboración es sencillamente menos problemática.

Aunque está claro que los problemas morales del médico son distintos a los del paciente, debido a que la responsabilidad del médico de llegar a un diagnóstico y u nrégimen curativo pesa mucho sobre su consciencia, el principio naturalista de que los médicos y los pacientes comparten fundamentalmente los mismos dilemas vitales tiende a reforzar la conexión entre la medicina y la vida del paciente considerada como un todo. Esto trae consigo una relación más abierta entre médico y paciente, y en el contexto de esta relación es más probable que el paciente encuentre el apoyo moral del médico en momentos de crisis.

Aunque la jerarquía de autoridad persiste en la medicina naturalista, las modalidades jerárquicas están menos marcadas y las semejanzas comunes a todos los seres humanos reciben un énfasis mayor. Esto afecta a las relaciones entre médico y paciente de una manera muy distinta a lo que hemos visto en medicina científica.

\section{La aplicación de los «ideal-types»}

Los perfiles de la medicina intervencionista y naturalista que acabamos de ver tienen cierta utilidad heurística. El espectro empírico de la medicina se extiende con grandes variantes y las diferencias entre las medicinas científica y naturalista pueden ser difíciles de definir en algunos casos concretos. La diversidad de las especialidades en la medicina científica, y la diversidad de tácticas curativas y tratamientos en la medicina naturalista se deben traer ahora a primer plano para poder así promulgar mejor la relación entre estas dos versiones de la medicina.

En los escritos antropológicos y sociológicos sobre medicina se ha hecho poco caso de la diversidad de la práctica médica dentro de la tradición científica. Las esferas de la oncología, la cardiología, la medicina interna, la pediatría, la ortopedia, la medicina pulmonar, la psiquiatría, etc., son excepcionalmente diversas, Las enfermedades, sus etiologías, sus efectos en los pacientes y los muchos tipos de tratamientos posibles, todo condiciona de un modo importante las relaciones entre médico y paciente. Dentro de la estructura de este artículo no me sería posible discutir todas estas diferencias, aunque estoy participando precisamente en una investigación de estas diferencias desde el punto de vista de la Antropología de la medicina (1984). Para ilustrar mi argumento resumiré brevemente los contrastes entre las especialidades de cardiología, terapéutica radiactiva o nuclear, y reumatología.

\section{Tres especialidades cientificas} pleja. Los pacientes llegan a los especialistas en cardiología sufriendo enfermedades cardíacas que pueden tener una evolución relativamente lenta o pueden adoptar el curso de un infarto repentino. Las técnicas de tratamiento cardiológico y de cirugía cardíaca son muy elaboradas. Los recursos tecnológicos de control y de evaluación, mediante monitores, que incluyen electrocardiogramas y cateterización cardíaca (técnica que permite filmar las contracciones del corazón y de los músculos cardíacos para determinar la presencia o estado de desarrollo de la enfermedad), las técnicas quirúrgicas de by-pass, la implantación de marcapasos y los complejos tratamientos farmacológicos hacen que este campo técnico requiera un alto nivel de entrenamiento y dedicación. El médico llega a tener prestigio tanto por su habilidad técnica como por su comportamiento frente al paciente.

En los casos caracterizados por un proceso lento, como la arteriosclerosis, la hipertensión y la angina de pecho recurrente, el médico entabla una relación a largo plazo con el paciente. El paciente experimenta la enfermedad como unos grados de disfunción y de limitación que van aumentando con el paso del tiempo. En el caso de los infartos de miocardio, los pacientes pueden despertarse de repente en la sala de cuidados intensivos del hospital bajo el cuidado de un cardiólogo, a quien tal vez no hayan conocido antes. A veces despiertan tras haber sido intervenidos quirúrgicamente o mientras les preparan para la cirugía. Los complejos instrumentos de control conectan también al paciente con el mundo de la tecnología. La relación entre médico y paciente, se desarrolla a posteriori.

Desde otro punto de vista, las enfermedades del corazón no tienden a ser conceptualizadas como una entidad que invade a la persona. En general se ven como una especie de fallo mecánico, independiente de la voluntad o condición moral del paciente. Este concepto mecanicista de las enferme- 
dades cardíacas se apoya en la compleja tecnología disponible: las posibi lidades de inserción de válvulas del corazón, la implantación de marca. pasos electrónicos y aun la posibilidad de trasplantes del órgano completo. Aunque las enfermedades del corazón no son por lo general de origen infeccioso, conducen a menudo a un alto nivel de disfunción para el paciente. Por lo general le causan un sentimiento de contaminación moral de un grado menor que otras enfermedades. Es la obligación del cardiólogo proporcionar un buen servicio técnico para que la máquina vuelva a funcionar bien. El mejor cardiólogo puede que sea el mejor «ingeniero»; de ahí que la presión para que el cardiólogo sea un individuo lleno de compasión no sea muy intensa.

En el servicio de terapia radiactiva, donde he empleado varias semanas de estudio, la escena es bastante distinta. Los pacientes que llegan a la misma tienen cánceres de diferentes tipos. En el caso del cáncer, la radiación tiene básicamente dos propósitos. En primer lugar, siempre hay cierta esperanza de que la terapia pueda controlar en parte el crecimiento de los tumores. En segundo, la radiación tiene además la capacidad de aliviar los fuertes dolores causados por los tumores localizados en distintas partes del cuerpo. Rara vez existe la posibilidad o la esperanza, tanto por parte del médico como del paciente, de que la terapia con radiación pueda proporcionar una cura definitiva. El paciente, desde el momento de su llegada, se define como un individuo enfermo. La terapia es principalmente paliativa, un intento de mejorar su existencia y de alargar en algo su vida.

En la mente popular no hay duda de que el cáncer es una enfermedad moralmente contaminadora. Tener cáncer, como nos ha demostrado tan convincentemente Susan Sontag (1978), es una experiencia aterradora. El cáncer es un asesino, un ente satánico, un contaminador; no respeta ni la edad ni la posición social. Es una sentencia de muerte. Todo ello conspira para colocar al paciente' en un estado de debilidad y contaminación. Una gran parte de la terapia se centra en el proceso por el cual el paciente aprende a vivir con su enfermedad y con la idea de que el cáncer es en fin de cuentas una sentencia de muerte.

En la terapia por radiación la tecnología disponible es realmente impresionante. Los potentes y complicados rayos láser se usan para dirigir la radiación con precisión absoluta sobre los tumores. Combinando los rayos $\mathrm{X}$ y la tomografía axial computerizada (TAC), el uso de ultrasonidos y la radiación, el radiólogo diseña un plan de ataque que incluye el número de tratamientos, la cantidad total de radiación que se piensa administrar, y una esterectaxia anatómica muy cuidadosa para localizar los puntos del cuerpo hacia donde debe dirigirse la radiación. La sala de radiación parece la cabina de una nave espacial en cuyo centro aparece una máquina tan grande que casi oculta al paciente. El médico y los técnicos preparan al paciente con mucho cuidado, dibujando sobre su cuerpo las coordenadas hacia donde dirigirán la radiación: a los tejidos invadidos por el cáncer, evitando tocar en lo posible los tejidos sanos.

El radiólogo es sólo uno entre el grupo de médicos que atienden a los pacientes de cáncer en el servicio de oncología. A pesar de la tecnología tan elaborada que lo rodea, la terapia por radiación en el hospital donde llevo a cabo mis investigaciones ofrece oportunidades para que los pacientes dialoguen con los médicos. Esto es debido al largo tiempo requerido para preparar a los pacientes que van a recibir la radiación. Durante estas preparaciones hablan de sus problemas, de sus preocupaciones, de sus expectativas vitales, y de otros problemas de una forma que no es posible en la sala de oncología donde comen y duermen. Es así que en medio de la tecnología más desarrollada existe un lugar apropiado para un tipo de diálogo terapéutico en el cual le es posible al médico simpatizar con el paciente. El entrenamiento técnico del médico, por lo tanto, no es la causa intrínseca de la enajenación entre médico y paciente. La tecnología de la era espacial que se asocia con la terapia por radiación no excluye automáticamente la interacción entre médico y paciente: esa interacción que uno esperaría encontrar más a menudo en la práctica de la medicina naturalista que en la de la científica.

Podemos ver un contraste aún más pronunciado observando la especialidad de reumatología. En esta especialidad los enfermos de artritis, dolores en las articulaciones y dolores difusos llegan al médico buscando un diagnóstico y tratamiento. La reumatología es un campo en el cual es excepcionalmente difícil diagnosticar. Aunque se dispone de análisis de sangre y otros tipos de análisis bioquímicos, y es posible asimismo valerse de los rayos $\mathrm{X}$, de los ultrasonidos y de las TAC; el núcleo del tratamiento reumatológico consiste en el proceso de preparar un detallado historial que ayude a enfocar bien los síntomas para llegar a una comprensión clara del tipo del dolor que el paciente experimenta y para juzgar su grado de disfunción. Pero sin el examen físico directo para ver cómo funcionan realmente las articulaciones de un paciente determinado, no se puede hacer nada en reumatología.

Llevándolo a un grado extremo, diríamos que el paciente de reumatología es una caja negra; porque nada en la tecnología diagnóstica proporciona al reumatólogo datos concluyentes. Esto hace que el especialista se vea obligado a mennudo a depender de las llamadas artes tradicionales de la medicina: la toma e interpretación del historial y el examen físico del 
paciente, para comprender a base de los signos externos lo que puede pasar dentro del paciente.

La anamnesis diagnóstica tiende a ser larga y detallada. El grado de manipulación del cuerpo que se necesita para examinar las articulaciones intensifica la relación personal entre el médico y el paciente. Además, muchas de las enfermedades tratadas por los reumatólogos son estados de enfermedad de larga duración y a menudo permanentes. Esto quiere decir que el reumatólogo verá a esos pacientes muchas veces a lo largo de su práctica y quiere también decir que los pacientes a menudo no esperan recobrar totalmente la salud, sino que tratan de mejorar la calidad de sus vidas lo suficiente como para mantener una actividad tolerable y productiva. No es raro que una gran parte del diálogo que se desarrolla en la práctica reumatológica se centre en establecer los parámetros de una calidad de vida razonable.

En reumatología, en consecuencia, a pesar de la existencia de una tecnología disponible, la relación entre médico y paciente se aproxima más al modelo de la medicina naturalista de lo que pudiéramos habernos imaginado. Es pues evidente que, al hablar de la medicina científica, es perentorio especificar el campo al cual nos referimos. Las enfermedades, la tecnología y los modos de recoger los diferentes datos; todo ello ejerce una influencia decisiva sobre la relación entre médico y paciente. Estas relaciones siguen un espectro que va desde aquéllas caracterizadas en mi ideal-type de la medicina científica, hasta las que se asemejan más a las relaciones descritas como tipicas de la medicina naturalista.

\section{Dos especialidades naturalistas:}

$\mathrm{Si}$ examinamos algunas variedades de la medicina naturalista, la necesidad de atender bien a la diversidad médica resulta igualmente patente. Podemos empezar por el polo más científico y técnico de la medicina naturalista, o sea la terapia por biofeedback. En la práctica de biofeedback se emplean aparatos electrónicos para controlar los distintos parámetros del estado fisiológico y neurológico del paciente. La actividad fisiológica y neurológica se muestra al paciente a través de monitores, y poco a poco se le enseña a controlar los procesos físicos que han degenerado en patología o que han quedado desequilibrados.

El énfasis central del biofeedback consiste en establecer o restaurar cierto tipo de equilibrio funcional. La terminología naturalista abunda en esta terapia. El concepto de equilibrio, la idea de autonomía del paciente y otros conceptos asociados colocan al práctico de esta terapia claramente dentro del contexto de la medicina naturalista. Sin embargo, un buen técnico de biofeedback utiliza la elaborada tecnología de la era espacial. Sin esta tecnología, la información sobre la cual se basa el tratamiento no estaría a la disposición consciente del paciente. Aquí pues tenemos el caso de un tipo de práctica médica que es a la vez naturalista y totalmente dependiente de la tecnología avanzada.

Otro nivel de práctica naturalista lo constituye la hidrología médica, es decir, el uso de las aguas minerales tanto en baños como en uso interno o como remedio curativo. La hidrología médica se basa en una complicada combinación de filosofía naturalista y teoría humoral. La premisa básica de la hidrología médica es que el funcionamiento interrumpido o alterado, cualquiera que sea su causa, pueda ser compensado por medio de los tratamientos hidrológicos. Para conseguir esto tiene que ser compensada la causa del desequilibrio y se receta una aplicación hidrológica capaz de restablecer el equilibrio. Los modos de aplicación de las aguas son distintos y muy variados. Existen las aspersiones, las aplicaciones de paños, varios tipos de masaje a chorro, la natación, baños de inmersión y de suspensión, masajes y una gran variedad de regímenes para consumir las aguas que forman parte de este tipo de tratamientos. Se usan masajes terapéuticos, se controla la dieta con cuidado y se orienta la organización completa de la vida diaria en el balneario para crear un tipo de régimen equilibrador.

Los tratados sobre hidrología médica son abundantes y complejos. Los análisis químicos de las aguas son detallados y la correlación entre las variadas propiedades químicas de las aguas y los diversos estados de enfermedad y trauma se desarrollan con gran minuciosidad. A pesar de toda esta elaboración, no se puede decir que este tipo de medicina demuestre un alto grado de sofisticación tecnológica. Aunque los análisis de las aguas han mejorado mucho con los nuevos recursos tecnológicos de análisis químico, y aunque existen más diseños expedimentales para comprobar la eficacia de los diversos tratamientos, el tratamiento básico mediante aguas es muy sencillo. El médico del balneario receta fundamentalmente regímenes. Analiza el problema del paciente, intenta descubrir qué tipo de desequilibrio lo ha causado, y luego, lentamente y sin invadir el cuerpo, crea un régimen capaz de volver al paciente a un grado de equilibrio o armonía funcional. Los tratamientos en sí no son ni intervencionistas ni drásticos. El paciente vuelve a ver al médico en un ciclo pre-establecido y el patrón del tratamiento se modifica de acuerdo con el tipo de progreso que experimenta el paciente.

En el balneario se trata a la persona total, porque el balneario es un medio ambiente total. El descanso, la vida social, la falta de tensión, y un 
refugio del mundo forman parte de la cura naturalista. Se supone que el estado moral del paciente mejora por el mero hecho de su presencia en el balneario; lo que demuestra que el régimen del balneario no se parece en nada al del hospital moderno.

Quedan muy claras las diferencias entre la medicina intervencionista y la medicina naturalista cuando se habla de cirugía cardíaca por un lado y de balneoterapia por el otro. Sin embargo, al considerar las otras especialidades médicas descritas en esta presentación resulta más difícil clasificarlas como intervencionistas o naturalistas. Está claro que la tecnología por sí sola no hace que la medicina sea científica, y la falta de tecnología tampoco convierte a la medicina naturalista en medicina intervencionista.

\section{Conclusiones}

Podemos concluir confesando que hacemos enormes generalizaciones en nuestros intentos de comprender la medicina científica. Nos hemos dejado impresionar por los cambios y transformaciones causados por los adelantos científicos y tecnológicos y tendemos a menospreciar el gran poder de ciertos conceptos de la salud y la enfermedad.

Los dilemas físicos y morales del paciente y las preocupaciones profesionales y personales del médico se entrelazan y condicionan de manera importante los resultados terapéuticos. El estudio de estas dimensiones socioculturales de la relación entre médico y paciente es una de las bases más importantes de la Antropología de la medicina. Algunos estados patológicos crean mayores dilemas morales que otros, pero todo tipo de enfermedad seria levanta consideraciones sobre la causa de sus aflicciones. Los problemas más difíciles en la relación entre médico y paciente ocurren en el polo extremo de la medicina científica, donde el médico intenta verse como puro científico, social e intelectualmente superior al paciente y sin responsabilidad alguna sobre su bienestar general. Pero esta posición extrema es bastante excepcional dentro de la medicina científica. No debe olvidarse tampoco que existen posibilidades de trato inhumano en la medicina naturalista. La técnica de biofeedback se puede reducir a rutinas insensatas, el tratamiento hidrológico puede ser un ejemplo del charlatanismo más descarado. A menudo las aplicaciones rutinarias de las aguas podrían ser sustituidas por una relación terapéutica con los mismos y mejores resultados. Es así que no hay tecnología ni tradición médica que pueda garantizar al paciente o al médico una solución satisfactoria.
También es necesario reconocer que todos los elementos de la medicina parecen estar presentes en todos los tipos de la práctica médica. La técnica y la tecnología, las faltas de simetría entre la experiencia social y en los conocimientos médicos de los médicos y pacientes, las dificultades del diagnóstico y el tratamiento, y los problemas del estado moral del médico y del paciente existen en todas las formas occidentales de la medicina. No nos debe sorprender pues que el llamado conflicto entre la medicina intervencionista y naturalista, en lugar de ser un rasgo típico de épocas históricas sucesivas, represente el desenvolvimiento cíclico de los elementos intrínsecos a la medicina occidental en sí.

He dado a esta presentación el subtítulo de «historia antropológica de una pugna ideológica», porque lo que he perfilado aquí es un acercamiento que requiere nuestra comprensión de los conflictos médicos contemporáneos como encarnaciones de problemas culturales mucho más antiguos y profundos dentro de la cultura occidental.

Una historia antropológica de la contienda entre estos dos tipos de medicina requiere por lo menos el análisis de la medicina como una construcción socio-cultural y como una colección de formas de conducta social. Existe una tendencia demasiado extendida a hacer hincapié exclusivamente en los factores sociales o en los culturales en cualquier explicación específica. Y hay tentación no menos fuerte a no examinar los detalles etnográficos del comportamiento médico. La medicina es más compleja, más diversa y más interesante de lo que parece en cualquiera de los tratados actuales sobre estos problemas.

Uno de los problemas más difíciles que tendremos que resolver como antropólogos interesados en la medicina occidental es el de reconocer la multifocalidad de los sistemas ideológicos. Tenemos una gran tendencia a tratar las ideologías como si fueran uniformes, insistiendo en saber si son jerárquicas o igualitarias, científicas o naturalistas, analíticas o integristas. Tal concepto de la ideología es demasiado pobre para ser útil en nuestras investigaciones. Elementos diversos, incluso opuestos, coexisten como partes de un solo sistema ideológico. En el proceso histórico hay un movimiento interno entre estos polos ideológicos. Yo aseguraría que en este caso la historia de la medicina nos demuestra cómo la medicina científica y la naturalista son realmente dos modalidades de un solo sistema ideológico. Seguir tal perspectiva nos impondrá la obligación de reorganizar radicalmente nuestra manera de considerar los sistemas ideológicos y su diversidad interna.

$\mathrm{Si}$ aceptamos este paso, los esfuerzos por una historia antropológica de la medicina se tendrán que dirigir a la investigación de los factores que mueven la ideología hacia una u otra de sus manifestaciones polarizadas en 
distintos momentos históricos. Tendremos que buscar las fuerzas que inclinan a la medicina hacia el polo intervencionista a veces y hacia el polo naturalista en otros momentos. El centro del análisis no deberá ponerse en la explicación de las raíces del intervencionismo ni del naturalismo, sino en la caracterización de los movimientos en la relación entre los dos vistos como una continuidad.

¿Por qué necesitamos una historia antropológica? Para empezar, porque es muy importante comprender la medicina moderna. La ciencia médica, los hospitales, el uso y abuso de drogas y la enorme industria sanitaria, constituyen uno de los factores más importantes de los presupuestos nacionales de la mayoría de los países desarrollados. Los problemas de la distribución de servicios sanitarios, las prioridades en la investigación y en el despliegue de los servicios médicos y la tecnología se encuentran entre los problemas nacionales más serios. No podemos estructurar sistemas médicos adecuados hasta que no comprendamos mejor la diversidad y las expectativas aparentemente contradictorias que guardamos respecto a la medicina. Es especialmente importante que lleguemos a equilibrar nuestras perspectivas sobre los sistemas socioculturales en la medicina. Los estudios de las instituciones médicas nacionales no se deben hacer en detrimento de los que consideran la medicina como un sistema de experiencias humanas.

La dimensión experiencial de la medicina, tanto para el médico como para el paciente, parece proporcionarnos información necesaria para nuestra comprensión del conflicto entre las distintas tradiciones médicas y nuestras metas contradictorias. La sociedad moderna ya ha empezado a oír las quejas de los pacientes porque llaman la atención con sus pleitos y las consecuencias económicas que eso trae. Pero, como en toda investigación antropológica, el problema está en saber qué significado tiene lo que dicen los miembros participantes.

\section{BIBLIOGRAFIA}

COULTER, Harris L.

Divided Legacy: A History of the Schism in Medical Thought, Volume III, Washington, D.C., McGrath Publishing Company.

1975 Divided Legacy, Volume I, Washington, D.C., Wehawken Book Co.

1977 Divided Legacy, Volume II, Washington, D.C., Wahawken Book Co.
FEIjó, Padre Benito Jerónimo de

1724-1739 Theatro crítico universal o discursos varios, en todo género de materias, para desengaño de errores comunes, 8 tomos, Madrid. Imprenta de Lorenzo Francisco Mojados.

FLEXNER, Abraham

1970 Medjcal Education in the United States and Canada, Washington, D.C., Carnegie Foundation for the Advancement of Teaching, edición facsímil por Science and Health Publications, Inc., Washington, D.C.

Foucault, Michel

1973 El nacimiento de la clinica, México. Siglo XIX.

GrEenWOOD, Davyd

ms The Taming of Darwinism: The persistence of non-evolutionary views in the study of humans, book-length MS.

1984 "La classificació , la incertitud mèdica i el curs moral del pacient: cap a una Antropologia de la Medicina» En: Comelles, J. M. (Comp.). Antropologia $i$ salut, Barcelona. Fundació Caixa de Pensions.

ILLICH, Ivan

1975 Medical Nemesis, London. Calder and. Boyars (trad. cast.).

KoHrman, Arthur

1979 «Bringing old arts to new sciences: A physician's perspective perspective", paper presented at the conference The humanistic disciplines in transition, Cornell University, November 29-December 1 .

Pelletier, Kenneth

1979 Holistic Medicine, New York, Delta/Seymour Lawrence.

REISER, Stanley Joel

1978 Medicine and the reign of technology, Cambridge, Cambridge UniSontag, Susan versity Press.

1977 - 1978 Illness as Metaphor, New York, Farrar, Strauss and Giroux. (Trad. cast. Barcelona, Munchnik, 1981.)

Torres Villarroel, Diego de

1794-1799 Obras completas, 15 tomos, Madrid: Imprenta de la viuda de

1970 Recetarios astrológico y alquímico, José Manuel Valles, editor, Ibarra. Madrid. Editora Nacional

WAITZKIN, Harold, and Barbara WATERMAN

1974 The exploitation of illness in capitalist society, Indianapolis, The Bobbs-Merrill Company, Inc. 